\title{
Toothpaste that features microrepair science
}

BioRepair from BlanX features microrepair science that actually penetrates and repairs microscopic holes in the tooth's enamel. Bio-active microcrystals bond with the teeth's natural dentine and form a protective layer over nerve endings within the teeth. BioRepair's formula allows you to repair daily wear caused by the acid in plaque, food and drink, before it can lead to decay.

Tooth enamel does not contain living cells and is not capable of regenerating. Therefore, any damage caused is irreversible. However, the hydroxyapatite microcrystals contained in BioRepair are virtually identical in chemical composition to the hydroxyapatite mineral that makes up dentine and tooth enamel.
The microcrystals can penetrate into the smallest cracks and flaws in teeth, where they dissolve and bind into the structure of the enamel and dentine to remineralise and repair the teeth.

This ability to remineralise hard tissues has an impact on several disorders. It can prevent cavities by filling lesions as well as protecting against sensitivity caused by erosion by covering over dentinal tubules. The apatite in the crystals can also absorb sulphate compounds such as H2S, which is responsible for halitosis.

The BioRepair from BlanX range includes Total Protection Toothpaste and Sensitivity Control Toothpaste.

Reader response number 50

\section{Dramatically reducing shrinkage}

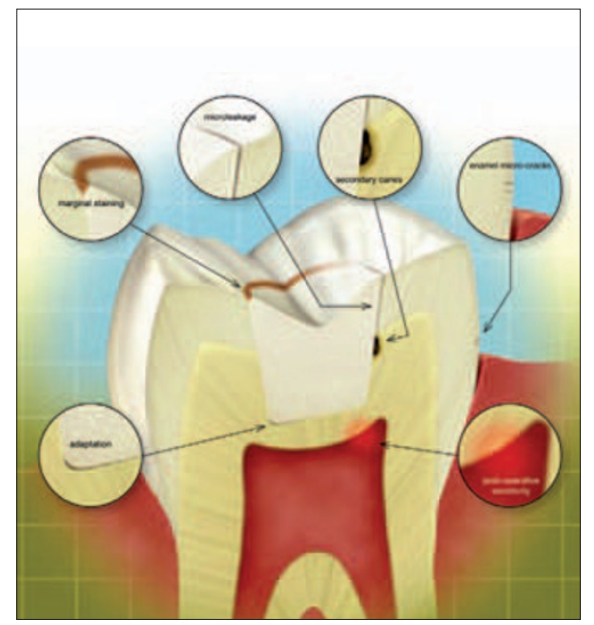

3M ESPE has launched a new posterior composite that shrinks less than $1 \%$. Filtek Silorane features 'ring-opening' chemistry which works at the molecular level to dramatically reduce shrinkage, compared to methacrylate-based composites.

As the silorane-based composite polymerises, ring-opening monomers connect by opening, flattening and extending toward each other. As methacrylate-based composites cure, the molecules of these linear monomers connect by actually shifting closer together in a linear response. The result is a loss of volume.

The new ring-opening polymerisation regime requires the use of a dedicated system adhesive: Silorane System Adhesive Self-Etch Primer and Bond. The self-etch adhesive is available in two vials which must be used consecutively; Silorane Self-Etch Primer used prior to Silorane Bond.

Combined with Silorane primer and bond, Filtek Silorane restorative provides a simple posterior restorative system for dentists who value low shrink technology. The complete restorative system features four popular radiopaque shades, in one opacity level and is delivered in capsules and syringes.

Reader response number 52 


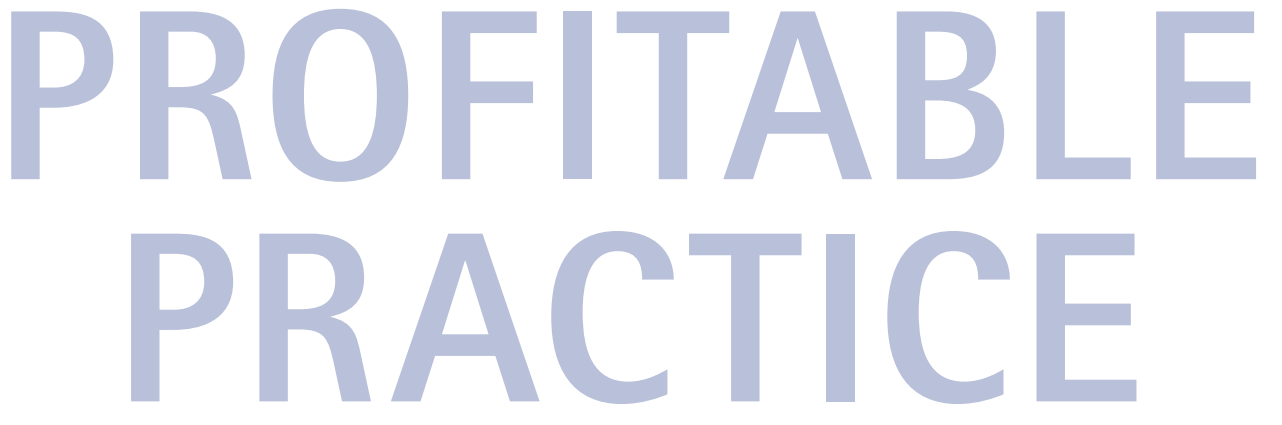

\section{Clean geometric lines}

Designer and manufacturer of surgery cabinetry Intercontidental has introduced its range Excel to the market. The range is characterised by clean geometric lines and hygienic glass or solid surface worktops.

Excel's work surfaces, in a choice of frosted or clear glass and solid surface colours, come with a choice of integral bowl and can be coupled with any suitable tap. The instrument drawers are very flexible, being offered in diverse heights and with easy to clean moulded, compartmentalised interior trays that are simple to remove for cleaning and have no square corners that can trap dirt and dust. The drawers glide effortlessly whilst cupboards are fitted with internal concealed UK electrical sockets, ideal for the connection of essential equipment.

The soft closing doors and drawer fronts are available in a choice of thirteen colours. All the materials used are

\section{Hard wearing cabinets}

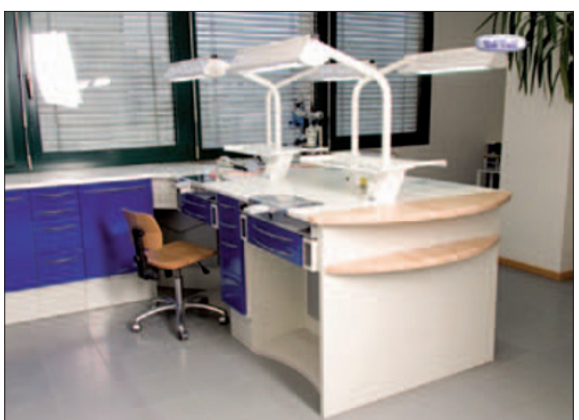

Tavom's hard wearing laboratory cabinets are uniquely created for each customer, based on individual needs and requirements. Work surfaces are tailor made to fit perfectly into any environment and sculptured to push the boundaries of style and elegance.

Its furniture is ideal for the dental laboratory, and Tavom UK's expert staff can advise on surprisingly cost effective solutions to improve working efficiency and enhance the working environment. Reader response number 54

\section{Selling your practice}

Frank Taylor \&t Associates, specialists in the sale of dental practices can alleviate the stresses and strains of selling your practice. All of your personal requirements are dealt with, while every aspect of the sale of your practice is negotiated and managed discreetly and professionally by the experts.

The company has a team of highly experienced valuers who will provide an independent valuation of your practice that reflects not only the physical value but also considers how established the practice is, as well as such things as staff and patient loyalty.

Reader response number 55

\section{Developing dental premises}

CareCapital is an expert developer of dental premises specialising in everything from allocating your ideal site, planning and financing, right through to design, construction and facilities management.

Whilst each individual project is tailored to suit your needs, the various steps involved in the development process commonly include briefing and business planning, site finding, programming and cost control.

By retaining the freehold, CareCapital is responsible for maintenance and upkeep, thus enabling the dentist to deliver high quality care in a customised surgery and premises without the additional burdens of owning the property.

This solution covers the entire development process, as well as providing a landlord-tenant relationship with a long-term view. In addition, CareCapital enjoys partnerships with many dental equipment suppliers enabling your dental facility to be uniquely tailored around your requirements and patient base, whether it is a single site practice, nationwide dental chain, or an institution such as a dental school.

Reader response number 56

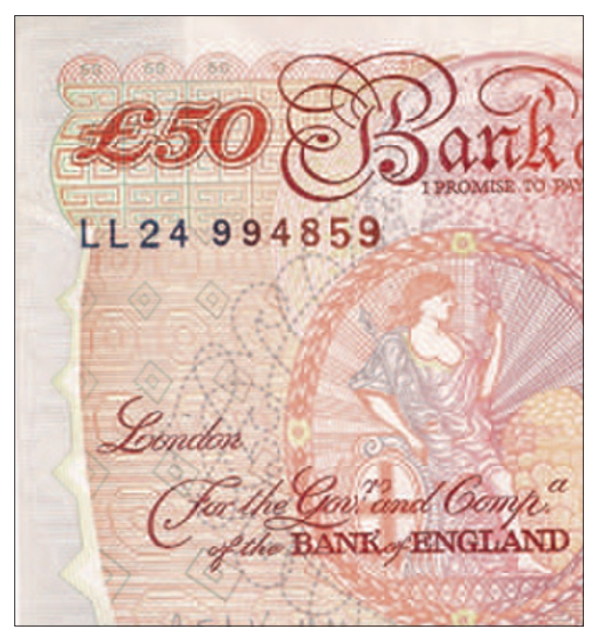




\section{Making your refurbishment profitable}

Takara Belmont, supplier of dental treatment centres, says it is essential to plan your refurbishment to make it pay as renewing your surgery equipment is one

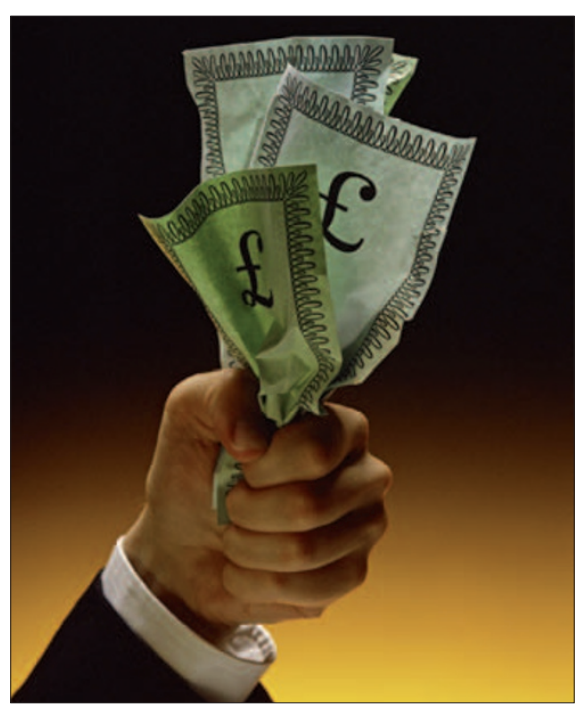

\section{Tailoring services to dentists}

The Association of Specialist Providers to Dentists (ASPD) is a group of companies located throughout the UK offering professional services adapted to the particular needs of dentists.

ASPD members include accountants, solicitors, banks, independent financial advisers, practice valuers and finance and leasing companies. Its primary objective is to provide dentists with reliable, practical advice canvassing all areas of their business, including selecting a bank sympathetic to the special requirements of a dental practice.

The ASPD's member banks employ highly trained dental specialists who are fully aware of the present industry background and can be relied upon to keep

\section{Financial advice}

Independent financial advisers money4 dentists have over 16 years experience behind them to exclusively advise dentists or partners of dentists. The company will help you to identify your objectives and priorities and guide you towards a practical, straightforward strategy to achieve your goals.

Matters such as residential mortgages or practice loans, private medical insurance, bank accounts, tax efficient savings, NHS pension scheme forecasts, private pension forecasts, education of the largest capital investments you can make.

It advises seeking advice from the experts and trying before you buy, in order to assess which equipment options exist and begin the search for what is the best practice solution. Timing your installation is also important, as is the choice of treatment centre.

All Belmont equipment can be preordered to your specification. Belmont's Clesta II has been designed with ease of installation very much in mind. It features a new aluminium base which reduces the weight of the unit without sacrificing stability, making it lighter and easier to install.

It also has a quick 'plug-in' Service Centre that minimises plumbing time and integrates all umbilical hoses into the low line base that helps create the clean lines of the Clesta II.

\section{Reader response number 57}

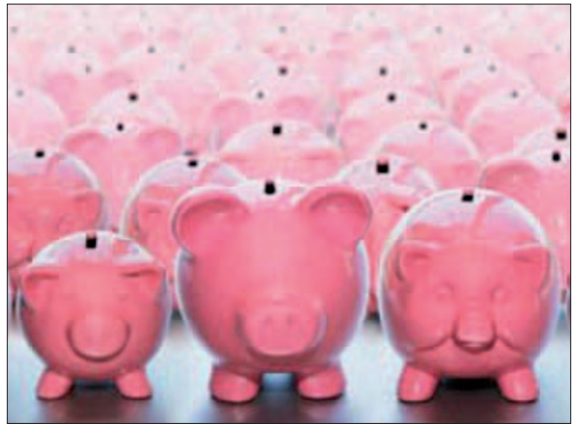

abreast of changes which may occur both within the dental profession and in the management or administration of the industry as a whole.

An ASPD bank member understands a practice's banking needs and can offer detailed advice on practical, efficient banking arrangements adapted to an individual practice.

Reader response number 58

plans or ensuring current investment funds reflect your current attitude to risk, are all key things a financial adviser can assist you with.

Reader response number 59

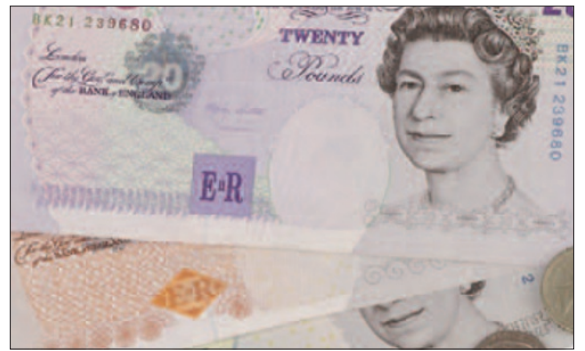

